\title{
Features of the effect of winter wheat selection on grain quality in the conditions of the South-Eastern region
}

\author{
Lyashcheva S.V.*, Kulevatova T.B. \\ Agricultural Research Institute of South-East Region, Saratov, Russia \\ *e-mail: lyaschevasveta@yandex.ru
}

Improving the quality of winter wheat grain is one of the priority areas of selection, whose efficiency is largely determined by source material. Evaluation of the quality of wheat grain may be different, depending on the field of its use. Grain quality is a set of technological and biochemical, baking and food properties, which determines the economic value of the variety. From the breeder's viewpoint, grain quality consists of many components which best correspond to the genetic basis of the varietal material which the breeding work is carried out with. Quality features, which are quantitative in nature, are very strongly influenced by environmental conditions. As a result of modifications, there may be situations when individuals with identical (by this or that sign) genotypes will be completely dissimilar relative to its phenotypic manifestation. When choosing the raw material for selection, one needs to take into account not only the genotypic potential of grain yield and quality, but also the degree of their preservation in adverse environmental conditions, the frequency of high-quality grain formation, and the reaction to changes in conditions during the formation and loading of grains. It is believed that in the arid conditions of the Volga region, the main limiting factor in the formation of high-quality grain is the amount of precipitation during the growing season of wheat and the uniformity of their distribution. More than 20 indicators of winter wheat quality are evaluated annually. With an ever-increasing amount of work on studying wheat quality in the selection process, the expression of many indicators through a smaller number is very important. Our study of the quantitative expression of the rheological properties of the dough on a Mixolab device makes it possible to study both the protein-proteinase complex and the carbohydrate-amylase one in one sample during one experiment. The data obtained indicate sufficient information content for selection (varietal diversity manifestation) of such indicators as water absorption capacity (WAC); the energy absorbed by dough during kneading (RA), dough stability, dough dilution (C2), and starch retrogradation index (C5). Dough formation time is the least informative. In $100 \%$ of cases, genotype-environmental interactions by this indicator were observed. The variation limits of the meal whiteness index was 5.914.6 units for the red wheat group and 14.3-28.1 for the white wheat group. The variation limits of the falling number in the white wheat group were $92-428 \mathrm{~s}$; the average value of this index was $248 \mathrm{~s}$. The variation limits of the falling number for the red wheat group were 290-382 s; the average value of this index was $341 \mathrm{~s}$. No relationship between the falling number and the meal whiteness index was proven: the correlation coefficient was 0.3482 only. 